\title{
Radiation Exposure of the Operator During Coronary Interventions (from the RADIO Study)
}

\author{
Zacharenia Kallinikou, MD ${ }^{\mathrm{a}}$, Serban G. Puricel, MD ${ }^{\mathrm{a}}$, Nick Ryckx, MSc ${ }^{\mathrm{b}}$, Mario Togni, MD ${ }^{\mathrm{a}}$, \\ Gerard Baeriswyl, MD ${ }^{\mathrm{a}}$, Jean-Christophe Stauffer, MD ${ }^{\mathrm{a}}$, Stephane Cook, MD ${ }^{\mathrm{a}}$, Francis R. Verdun, $\mathrm{PhD}^{\mathrm{b}}$, \\ and Jean-Jacques Goy, MD ${ }^{\mathrm{a}, *}$
}

\begin{abstract}
We sought to compare operator radiation exposure during procedures using right femoral access (RFA), right radial access (RRA), and left radial access (LRA) during coronary angiography (CA) and percutaneous coronary intervention (PCI). Because of an increased incidence of long-term malignancy in interventional cardiologists, operator radiation exposure is of rising concern. This prospective study included all consecutive patients who underwent elective or emergency CA \pm PCI from September 2014 to March 2015. The primary end point was operator radiation exposure, quantified as the ratio of operator cumulative dose (CD) and patient radiation reported as dose-area product (DAP) (CD/DAP). Secondary end points included CD, DAP, and fluoroscopy time (FT). Overall 830 procedures (457 CA [55\%] and $373 \mathrm{PCI}$ [45\%]) were performed, $455(55 \%)$ through RFA, $272(33 \%)$ through RRA, and $103(12 \%)$ through LRA. The CD/DAP was lower in RFA $\left(0.09 \mu \mathrm{Sv} / \mathrm{Gycm}^{2}\right.$ [0.02 to 0.20$\left.]\right)$ compared with RRA $\left(0.47 \mu \mathrm{Sv} / \mathrm{Gycm}^{2}\right.$ [0.25 to 0.75$]$, p <0.001). The LRA showed lower CD/DAP compared with RRA ( $<<0.001)$. CD was significantly lower in RFA ( $3 \mu \mathrm{Sv}$ [1 to 7]) compared with RRA (12 $\mu \mathrm{Sv}$ [6 to 29], $\mathrm{p}<0.001)$. The LRA showed lower CD compared with RRA $(p<0.001)$. There were no significant differences in DAP among the 3 access sites. FT was similar for the 3 groups (RFA $7 \pm 7$, RRA $5 \pm 5$, LRA $6 \pm 5$ minutes, RFA vs RRA: $p=1$, RFA vs LRA: $p=0.16$, RRA vs LRA: $p=0.52$ ). In conclusion, the use of RFA during CA \pm PCI is associated with significantly lower operator radiation exposure compared with RRA. LRA is associated with significantly lower operator radiation exposure compared with RRA.
\end{abstract}

Because of a presumably increased stochastic risk of cancer induction among interventional cardiologists, especially neoplasms of the unprotected brain, nasopharyngeal tract, and upper extremities, operator radiation exposure during coronary angiography (CA) and/or percutaneous coronary intervention (PCI) is of rising concern. ${ }^{1,2}$ Therefore, we undertook a comparison of operator radiation exposure during right femoral access (RFA), left radial access (LRA), and right radial access (RRA) during CA and CA followed by ad hoc PCI in a real-world population.

\section{Methods}

From September 2014 to March 2015 at the University and Hospital Fribourg, all consecutive procedures of elective or emergency CA and CA followed by ad hoc PCI were prospectively considered for operator radiation exposure measurements. Procedures were performed by 5 senior

${ }^{a}$ Department of Cardiology, Hospital and University of Fribourg, Fribourg, Switzerland; and ${ }^{\mathrm{b}}$ Department of Radiology, Institute of Radiophysics, Centre Hospitalier Universitaire Vaudois, Lausanne, Switzerland. Manuscript received January 5, 2016; revised manuscript received and accepted April 26, 2016.

See page 194 for disclosure information.

*Corresponding author: Tel: (+41) 26426 7285; fax: (+41) 26 4268135 .

E-mail address: jjgoy@goyman.com (J.-J. Goy). interventional cardiologists with significant experience $(>3,000 \mathrm{PCI}$ each) in both femoral and radial access routes. Selection of the percutaneous access site was left to the discretion of the operator. Crossover access site procedures were excluded. This study was part of the Catheterization Registry Fribourg (CardioFR), which was approved by the Ethics Committee of Canton Vaud (protocol no: 339/14).

The primary end point of the study was operator radiation exposure, expressed as the cumulative equivalent dose (in $\mu \mathrm{Sv}$ ) over the lead apron at chest level, normalized for the patient radiation exposure (dose-area product [DAP] in $\mathrm{Gycm}^{2}$ ). Secondary end points included cumulative dose (CD), DAP, and fluoroscopy time (FT).

Procedures were performed on a digital single-plane cineangiography unit (Allura FD10; Philips Medical Systems, Hamburg, Germany) with an undertable x-ray tube MRC20025 with a magnification factor leading to a field of view of $21 \mathrm{~cm}$ and an acquisition frequency of 15 frames/s. All procedures were performed with respect to current guidelines using either $5 \mathrm{Fr}$ or $6 \mathrm{Fr}$ hydrophilic sheaths. Conventional diagnostic and guiding catheters were used.

The femoral access was achieved, under local anesthesia with $2 \%$ rapidocain, through the anterior wall puncture of the artery; 5Fr or 6Fr Terumo (Pinnacle; Terumo Medical, Tokyo, Japan) introducer was placed in the femoral artery. $\mathrm{CA}$ and PCI were performed according to standard practice using catheter and drugs left to the discretion of the operator. Hemostasis was achieved using closure devices 


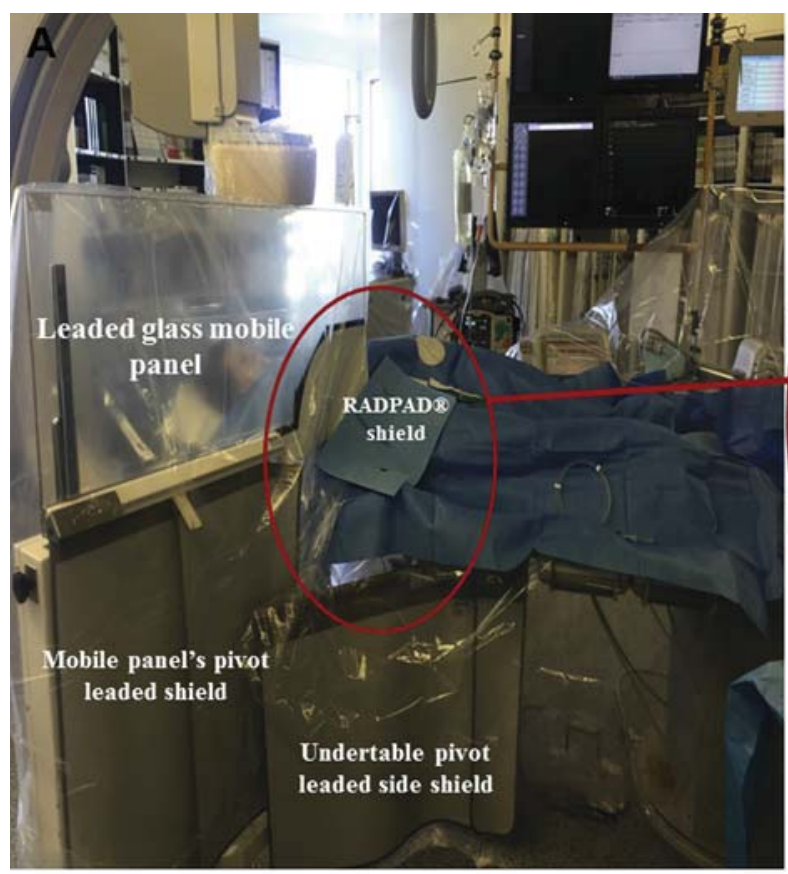

\section{B}

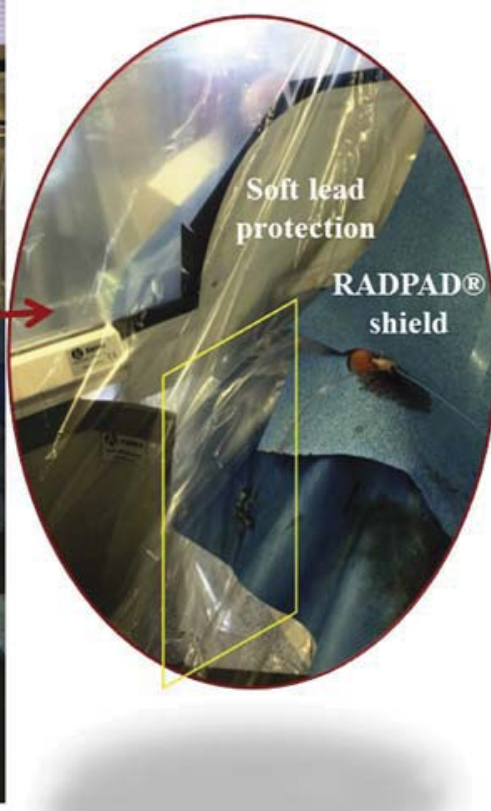

Figure 1. (A) Radioprotection equipment and materials. Image acquisition during a right radial case. (B) Although the shields are approximated as closely as possible to minimize operator irradiation, there is still a radioprotection gap (yellow box), which is inevitably more important during right radial procedures.

Table 1

Baseline patient and procedural characteristics

\begin{tabular}{|c|c|c|c|c|c|c|}
\hline & \multirow[t]{2}{*}{ RFA $(\mathrm{N}=455)$} & \multirow{2}{*}{ LRA $(\mathrm{N}=103)$} & \multirow{2}{*}{ RRA $(N=272)$} & \multicolumn{3}{|c|}{$\mathrm{p}$-values } \\
\hline & & & & RFA vs. LRA & RFA vs. RRA & LRA vs. RRA \\
\hline \multicolumn{7}{|l|}{ Patients } \\
\hline Age (years $\pm \mathrm{SD})$ & $68 \pm 12$ & $69 \pm 10$ & $65 \pm 12$ & 1.00 & 0.02 & 0.05 \\
\hline Male & $310(68 \%)$ & $71(69 \%)$ & $181(67 \%)$ & 1.00 & 1.00 & 1.00 \\
\hline Weight $(\mathrm{kg} \pm \mathrm{SD})$ & $80 \pm 16$ & $80 \pm 18$ & $81 \pm 16$ & 1.00 & 1.00 & 1.00 \\
\hline Height $(\mathrm{m} \pm \mathrm{SD})$ & $1.70 \pm 0.09$ & $1.70 \pm 0.09$ & $1.70 \pm 0.09$ & 1.00 & 1.00 & 1.00 \\
\hline $\mathrm{BMI}\left(\mathrm{kg} / \mathrm{m}^{2} \pm \mathrm{SD}\right)$ & $27 \pm 5$ & $27 \pm 5$ & $28 \pm 5$ & 1.00 & 1.00 & 1.00 \\
\hline \multicolumn{7}{|l|}{ Procedure } \\
\hline $\mathrm{CA}$ & $216(47 \%)$ & $65(63 \%)$ & $176(65 \%)$ & 0.01 & $<0.01$ & 1.00 \\
\hline CA followed by ad hoc PCI & $239(53 \%)$ & $38(37 \%)$ & $96(35 \%)$ & 0.01 & $<0.01$ & 1.00 \\
\hline Procedural time $(\min \pm \mathrm{SD})$ & $20 \pm 19$ & $15 \pm 11$ & $18 \pm 12$ & 0.05 & 1.00 & 0.08 \\
\hline Fluroscopy time $(\min \pm \mathrm{SD})$ & $7 \pm 7$ & $5 \pm 5$ & $6 \pm 5$ & 0.16 & 1.00 & 0.52 \\
\hline $\mathrm{Nb}$ of cine-angiograms $(\mathrm{n} \pm \mathrm{SD})$ & $954 \pm 520$ & $659 \pm 351$ & $727 \pm 300$ & $<0.001$ & $<0.01$ & $<0.001$ \\
\hline
\end{tabular}

Variables are reported in numbers $(\%)$ or mean $\pm \mathrm{SD}$.

$\mathrm{BMI}=$ body mass index $\mathrm{CA}=$ coronary angiography; DAP $=$ dose area product; $\mathrm{LRA}=$ left radial access; PCI $=$ percutaneous coronary intervention; $\mathrm{RFA}=$ right femoral access; RRA $=$ right radial access; $\mathrm{SD}=$ standard deviation.

(Femoseal; St. Jude Medical, St. Paul, Minnesota) or external mechanical compression (Femostop; St. Jude Medical).

After sterile preparation and anesthesia with $2 \%$ rapidocain infiltration, radial artery was punctured with a 20gauge needle. A 0.021 Teflon-sheathed short guidewire was inserted in the needle. A 3.2-section BD Venflon was then inserted in the artery. A Terumo (Pinnacle; Terumo Medical) 0.021 hydrophilic guidewire was advanced through the radial and brachial artery. A 5Fr introducer was then inserted in the radial artery. Vasodilator cocktail consisting of verapamil $3 \mathrm{mg}$ and enoxaparin 3,400 units was administered after sheath insertion. Specific catheters were used for CA and PCI. Exchange to a 6Fr sheath was possible when technically necessary. At the end of the procedure, the sheath was removed and an inflatable pressure band placed to the access site. All radial accesses were performed at the right side of the patient.

Operator protection was ensured with the same equipment for all procedures. A leaded glass mobile panel with a 


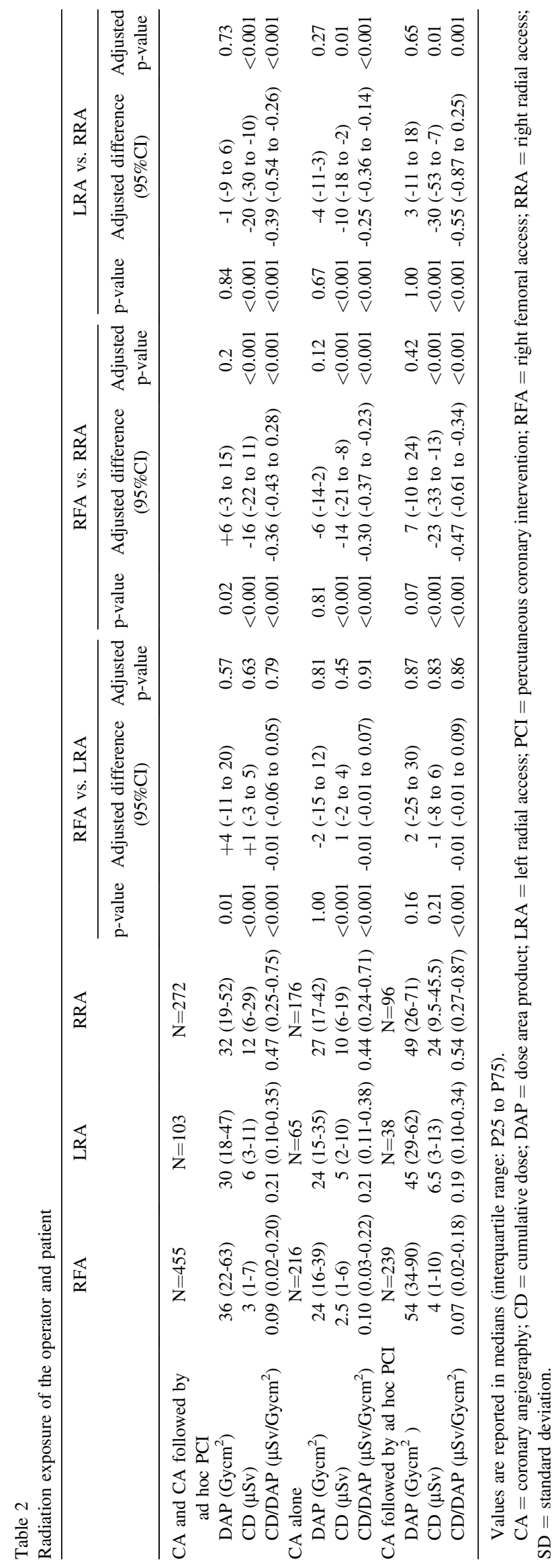

patient contour cutout (0.5 lead equivalent; MAVIG, Munich, Germany) was positioned at the left side of the operator. An undertable pivot-leaded side shield $(0.5-\mathrm{mm}$ lead equivalent) was mounted to the side of the table. An additional soft lead shield was adjusted in the contour cut of the leaded glass mobile panel to minimize radiation exposure. The $37 \times 42 \mathrm{~cm}$ upper-shield flap (RADPAD, Worldwide Innovations \& Technologies, Inc, Kansas City, USA) was placed over the access site in each procedure to reduce scatter radiation (Figure 1). Additional radiation protection materials were standardized for all operators and included a lead apron, thyroid lead collar, and leaded glasses. All procedures were performed from the patients' anatomical right side.

Operator radiation was measured using individual electronic dosimeters (DoseAware; Philips Healthcare, Best, The Netherlands) positioned on the sternum, outside the lead apron. The dosimeters are silicon-based semiconductor detectors with a dose-response between $1 \mu \mathrm{Sv}$ and $10 \mathrm{~Sv}$, in steps of $1 \mu \mathrm{Sv}$ (calibrated in ambient equivalent dose $\mathrm{Hp}(10)$ ) and a temporal resolution of 1 second. The following parameters were recorded for each procedure: (1) operator CD through the use of dedicated readout software (DoseView), measured by the individual dosimeters; (2) FT; (3) number of cine angiograms (NC); and (4) the DAP-normalized CD defined as the dose ( $\mu \mathrm{Sv})$ received by the operator with each $\mathrm{Gycm}^{2}$ applied to the patient (known as the exposure factor) has been advocated $^{3}$ and applied to our study as it isolates differences in patient radiation among the 3 vascular access sites. Patient radiation dose was expressed as DAP. Furthermore, radiation exposure of the assistant nurse, the first nurse on the operator's right side, was assessed using similar dosimeters, in a subgroup of consecutive procedures, with the aim to compare radiation exposure of the operator versus assistant nurse.

All statistical analyses were performed using dedicated software (Stata, version 13; StataCorp LP, College Station, Texas) at a 2-tailed significance level of alpha $<0.05$. Baseline patient and procedural characteristics, and variables assessing radiation exposure of the operator, were compared among the 3 vascular access sites. Categorical variables are reported as counts and percentages; continuous variables are reported as mean and SD or as median with $25 \%$ to $75 \%$ interquartile range according to their distribution. Normality was assessed by visual inspection of histograms, the computation of Q-Q plots and the Shapiro-Wilk test. Categorical variables were compared using the chi-square or Fisher's exact test as appropriate. Continuous variables were analyzed using the 1-way ANOVA or the Wilcoxon rank-sum test according to their distribution. To account for differences of the individual operators on radiation exposure according to access site, we computed a generalized linear model including the individual operators as potential confounders of the overall treatment effect. To account for multiple comparisons, $\mathrm{p}$ values are Bonferroni adjusted, that is, multiplied by the number of comparisons. Comparison of radiation exposure between the operator and the first assistant was assessed using the paired student's $t$ test or the signed-rank Wilcoxon test according to distribution. 

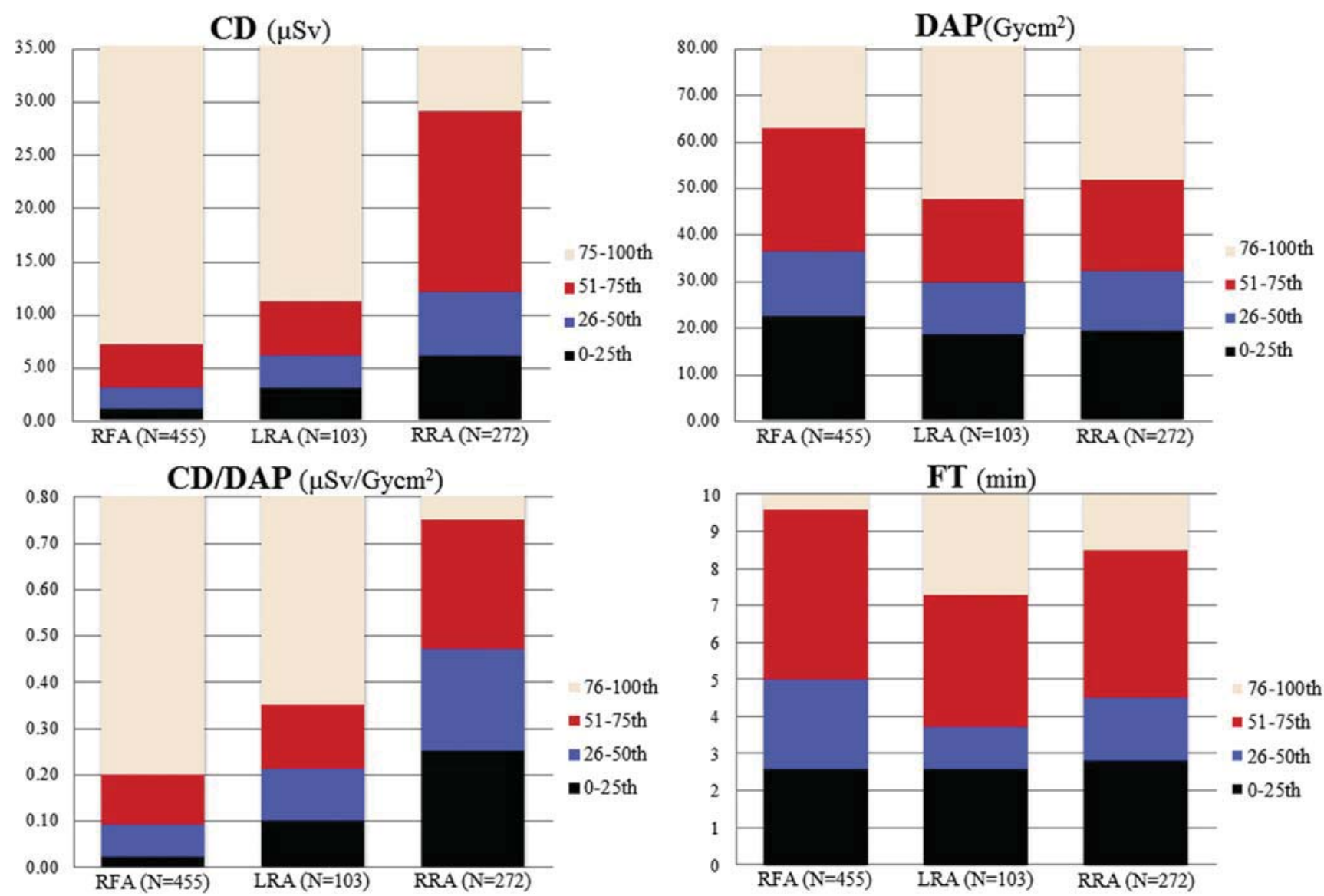

Figure 2. Operator CD, patient radiation dose (DAP), operator radiation exposure (CD/DAP), and FT for all procedures: CA and CA followed by ad hoc PCI. Columns: 3 vascular access sites in percentiles (colors): black: 0 to 25 th, blue: 26 to 50th, red: 51 to 75 th, and beige: 76 to 100 th, median values on the board of blue and red columns.

\section{Results}

During September 2014 and March 2015, 849 consecutive procedures for elective or emergency CA and CA followed by ad hoc PCI were performed in our catheterization laboratory with a dedicated dosimeter for radiation evaluation. Nineteen procedures $(1.8 \%)$ were excluded because of a crossover in access sites. No patient was excluded because of lesion or procedural complexity. Finally, 830 consecutive procedures were included in the trial, $457 \mathrm{CA}$ alone (55\%) and $373 \mathrm{CA}$ followed by ad hoc PCI $(45 \%)$. With regard to vascular site, 455 procedures $(55 \%)$ were carried out using the RFA, $103(12 \%)$ using the LRA, and 272 (33\%) using the RRA. Baseline patient characteristics were generally well balanced and depicted in Table 1. Analysis of procedural characteristics as listed in Table 1 revealed no difference in FT among the groups and showed a significantly greater NC in RFA compared with LRA and RRA group and in RRA compared with the LRA group, probably reflecting higher procedural complexity.

The radiation exposure of the assistant nurse standing at the operator's right side was also assessed in a subgroup of first 293 consecutive procedures, $152 \mathrm{CA}$ alone (52\%) and 141 CA followed by ad hoc PCI (48\%), $164(56 \%)$ through RFA, $49(17 \%)$ through LRA, and $80(27 \%)$ through RRA.
Table 2 and Figure 2 list patient and operator radiation exposure. Adjusted CD was significantly lower in RFA compared with RRA but not to LRA. CD was lower for procedures using LRA than RRA. Patient radiation dose expressed by DAP did not significantly vary among the 3 access sites. Operator radiation dose reported as the DAPnormalized $C D$ was significantly higher in the RRA compared with the RFA and LRA group for all procedures, procedures with $\mathrm{CA}$ alone, and procedures with CA followed by ad hoc PCI. There were no significant differences between the RFA and the LRA for any kind of procedure. Table 3 indicates DAP, CD, and DAPnormalized $\mathrm{CD}$ for the individual operators. A significantly higher radiation exposure comparing the RRA with the RFA was consistently found for all operators. Significant differences in radiation exposure between RFA and LRA and RRA and LRA were found only for 2 of the 5 operators. Radiation exposure for elective and urgent procedures is provided in Table 4. During elective procedures, radiation exposure was lower for RFA compared with RRA, but not to LRA. However, LRA showed a lower DAP-normalized CD compared with RRA $(\mathrm{p}<0.001)$. In addition to the aforementioned differences, LRA was associated with a higher radiation exposure than RFA, when only urgent procedures were considered 
Table 3

Radiation exposure according to the individual operator

\begin{tabular}{|c|c|c|c|c|c|c|}
\hline & \multirow[t]{2}{*}{ RFA } & \multirow[t]{2}{*}{ LRA } & \multirow[t]{2}{*}{ RRA } & \multicolumn{3}{|c|}{ p-values } \\
\hline & & & & RFA vs. LRA & RFA vs. RRA & LRA vs. RRA \\
\hline \multicolumn{7}{|c|}{ CA andCA followed by ad hoc PCI } \\
\hline Operator 1 & $\mathrm{~N}=39$ & $\mathrm{~N}=77$ & $\mathrm{~N}=88$ & & & \\
\hline DAP $\left(\mathrm{Gycm}^{2}\right)$ & $31(20-59)$ & $28(17-41)$ & $32(19-55)$ & 0.28 & 1.00 & 0.16 \\
\hline $\mathrm{CD}(\mu \mathrm{Sv})$ & $9(4-15)$ & $6(4-11)$ & $15.5(8-33)$ & 0.08 & $<0.01$ & $<0.001$ \\
\hline $\mathrm{CD} / \mathrm{DAP}\left(\mu \mathrm{Sv} / \mathrm{Gycm}^{2}\right)$ & $0.28(0.16-0.44)$ & $0.23(0.14-0.35)$ & $0.51(0.37-0.84)$ & 0.33 & $<0.001$ & $<0.001$ \\
\hline Operator 2 & $\mathrm{~N}=132$ & $\mathrm{~N}=3$ & $\mathrm{~N}=28$ & & & \\
\hline DAP $\left(\mathrm{Gycm}^{2}\right)$ & $44(31-90)$ & $67(37-90)$ & $36(18-56)$ & 1.00 & 0.07 & 0.20 \\
\hline $\mathrm{CD}(\mu \mathrm{Sv})$ & $1(0-2)$ & $1(0-3)$ & $1(0.5-7.5)$ & 1.00 & $<0.01$ & 1.00 \\
\hline $\mathrm{CD} / \mathrm{DAP}\left(\mu \mathrm{Sv} / \mathrm{Gycm}^{2}\right)$ & $0.01(0-0.04)$ & $0.01(0.00-0.04)$ & $0.05(0.00-0.17)$ & 1.00 & $<0.01$ & 0.53 \\
\hline Operator 3 & $\mathrm{~N}=98$ & $\mathrm{~N}=1$ & $\mathrm{~N}=3$ & & & \\
\hline DAP $\left(\mathrm{Gycm}^{2}\right)$ & $36(21-67)$ & 73 & $135(14-166)$ & 0.73 & 0.60 & 1.00 \\
\hline $\mathrm{CD}(\mu \mathrm{Sv})$ & $4(2-10)$ & 7 & $87(25-484)$ & 1.00 & $<0.01$ & 0.02 \\
\hline $\mathrm{CD} / \mathrm{DAP}\left(\mu \mathrm{Sv} / \mathrm{Gycm}^{2}\right)$ & $0.10(0.05-0.19)$ & 0.09 & $1.78(0.64-2.90)$ & 1.00 & $<0.01$ & 0.36 \\
\hline DAP $\left(\mathrm{Gycm}^{2}\right)$ & $30(17-54)$ & $36(24-128)$ & $33(22-50)$ & 0.23 & 0.49 & 0.82 \\
\hline $\mathrm{CD}(\mu \mathrm{Sv})$ & $4(1-6)$ & $1.5(1-3.5)$ & $12(7-36)$ & 0.16 & $<0.001$ & $<0.001$ \\
\hline $\mathrm{CD} / \mathrm{DAP}\left(\mu \mathrm{Sv} / \mathrm{Gycm}^{2}\right)$ & $0.10(0.05-0.18)$ & $0.05(0.01-0.08)$ & $0.45(0.24-0.78)$ & $<0.01$ & $<0.001$ & $<0.001$ \\
\hline Operator 5 & $\mathrm{~N}=47$ & $\mathrm{~N}=10$ & $\mathrm{~N}=84$ & & & \\
\hline DAP $\left(\mathrm{Gycm}^{2}\right)$ & $35(21-51)$ & $27(14-49)$ & $30(17-47)$ & 0.92 & 0.40 & 1.00 \\
\hline $\mathrm{CD}(\mu \mathrm{Sv})$ & $7(4-13)$ & $12(11-37)$ & $12(8-28)$ & 0.04 & $<0.001$ & 0.81 \\
\hline CD/DAP $\left(\mu \mathrm{Sv} / \mathrm{Gycm}^{2}\right)$ & $0.21(0.12-0.35)$ & $0.59(0.44-1.43)$ & $0.48(0.32-0.72)$ & $<0.01$ & $<0.001$ & 0.55 \\
\hline
\end{tabular}

Values are reported in medians (interquartile range: $\mathrm{P} 25$ to $\mathrm{P} 75)$.

$\mathrm{CA}=$ coronary angiography; $\mathrm{CD}=$ cumulative dose DAP $=$ dose area product; LRA = left radial access; PCI = percutaneous coronary intervention; $\mathrm{RFA}=$ right femoral access; RRA $=$ right radial access; $\mathrm{SD}=$ standard deviation.

Table 4

Radiation exposure of the operator and patient stratified by operator

\begin{tabular}{|c|c|c|c|c|c|c|c|c|c|}
\hline & \multirow[t]{2}{*}{ RFA } & \multirow[t]{2}{*}{ LRA } & \multirow[t]{2}{*}{ RRA } & \multicolumn{3}{|c|}{ Crude p-values } & \multicolumn{3}{|c|}{ Adjusted p-values } \\
\hline & & & & $\begin{array}{c}\text { RFA vs. } \\
\text { LRA }\end{array}$ & $\begin{array}{c}\text { RFA vs. } \\
\text { RRA }\end{array}$ & $\begin{array}{c}\text { LRA vs. } \\
\text { RRA }\end{array}$ & $\begin{array}{c}\text { RFA vs. } \\
\text { LRA }\end{array}$ & $\begin{array}{c}\text { RFA vs. } \\
\text { RRA }\end{array}$ & LRA vs. RRA \\
\hline \multicolumn{10}{|c|}{ CA and CA followed by ad hoc PCI } \\
\hline Elective & $\mathrm{N}=285$ & $\mathrm{~N}=90$ & $\mathrm{~N}=206$ & & & & & & \\
\hline DAP $\left(\mathrm{Gycm}^{2}\right)$ & $34(21-57)$ & $32(19-48)$ & $31(19-50)$ & 0.37 & 0.41 & 1.00 & 0.77 & 0.35 & 0.72 \\
\hline $\mathrm{CD}(\mu \mathrm{Sv})$ & $3(1-6)$ & $6(3-12)$ & $11(6-25)$ & $<0.001$ & $<0.001$ & $<0.001$ & 0.36 & $<0.001$ & $<0.01$ \\
\hline CD/DAP $\left(\mu \mathrm{Sv} / \mathrm{Gycm}^{2}\right)$ & $0.09(0.03-0.20)$ & $0.22(0.12-0.35)$ & $0.43(0.23-0.72)$ & $<0.001$ & $<0.001$ & $<0.001$ & 0.13 & $<0.001$ & $<0.001$ \\
\hline Urgent & $\mathrm{N}=170$ & $\mathrm{~N}=13$ & $\mathrm{~N}=66$ & & & & & & \\
\hline DAP $\left(\mathrm{Gycm}^{2}\right)$ & $41(25-84)$ & $25(12-44)$ & $38(21-57)$ & 0.02 & 0.11 & 0.29 & 0.69 & 0.62 & 0.16 \\
\hline $\mathrm{CD}(\mu \mathrm{Sv})$ & $3(1-10)$ & $4(1-6)$ & $17.5(8-41)$ & 1.00 & $<0.001$ & $<0.001$ & $<0.01$ & $<0.001$ & 0.001 \\
\hline $\mathrm{CD} / \mathrm{DAP}\left(\mu \mathrm{Sv} / \mathrm{Gycm}^{2}\right)$ & $0.08(0.02-0.19)$ & $0.15(0.10-0.21)$ & $0.55(0.37-0.85)$ & 0.16 & $<0.001$ & $<0.001$ & $<0.01$ & $<0.001$ & $<0.001$ \\
\hline
\end{tabular}

Values are reported in medians (interquartile range: P25 to P75).

$\mathrm{CA}=$ coronary angiography $\mathrm{CD}=$ cumulative dose; $\mathrm{DAP}=$ dose area product; LRA $=$ left radial access; PCI $=$ percutaneous coronary intervention; $\mathrm{RFA}=$ right femoral access; RRA $=$ right radial access; $\mathrm{SD}=$ standard deviation.

$(\mathrm{p}<0.01)$. In the subgroup of the first consecutive 293 procedures, operator exposure was significantly higher compared with the assistant nurse's as listed in Table 5 for all procedures and by access site.

\section{Discussion}

The main findings of the current prospective trial are the following: (1) interventional Cardiologists are exposed to a lower degree of radiation when performing CA or CA followed by ad hoc PCI through the RFA rather than the RRA; (2) interventional cardiologists are exposed to a lower degree of radiation when performing CA or CA followed by ad hoc PCI using the LRA rather than the RRA; and (3) the operator is more exposed to radiation compared with the assistant nurse standing at his right side during the procedures.

Transradial cardiac catheterization is known to be associated with an increased operator radiation dose even for highly experienced interventional cardiologists and despite the use of radioprotection optimization techniques. ${ }^{4-9}$ Data are limited with regard to operator radiation exposure when right and left radial accesses are compared, ${ }^{10-13}$ with most investigators reporting lower radiation levels for LRA, alike 
Table 5

Comparison of radiation exposure between operator and assistant nurse

\begin{tabular}{|c|c|c|c|}
\hline $\begin{array}{l}\text { CA and CA followed by } \\
\text { ad hoc PCI }(N=293)\end{array}$ & Operator & Assisstant nurse & p-value \\
\hline $\mathrm{DAP}\left(\mathrm{Gycm}^{2}\right)$ & $32(20-53)$ & $32(20-53)$ & 1.00 \\
\hline $\mathrm{CD}(\mu \mathrm{Sv})$ & $5(1-13)$ & $2(1-5)$ & $<0.001$ \\
\hline CD/DAP $\left(\mu \mathrm{Sv} / \mathrm{Gycm}^{2}\right)$ & $0.16(0.04-0.46)$ & $0.07(0.03-0.13)$ & $<0.001$ \\
\hline \multicolumn{4}{|l|}{ RFA $(\mathrm{N}=164)$} \\
\hline $\mathrm{CD} / \mathrm{DAP}\left(\mu \mathrm{Sv} / \mathrm{Gycm}^{2}\right)$ & $0.06(0.01-0.18)$ & $0.06(0.03-0.11)$ & $<0.05$ \\
\hline \multicolumn{4}{|l|}{ LRA $(\mathrm{N}=49)$} \\
\hline $\mathrm{CD} / \mathrm{DAP}\left(\mu \mathrm{Sv} / \mathrm{Gycm}^{2}\right)$ & $0.23(0.10-0.45)$ & $0.06(0.04-0.10)$ & $<0.001$ \\
\hline \multicolumn{4}{|l|}{ RRA $(\mathrm{N}=80)$} \\
\hline $\mathrm{CD} / \mathrm{DAP}\left(\mu \mathrm{Sv} / \mathrm{Gycm}^{2}\right)$ & $0.51(0.32-0.81)$ & $0.10(0.06-0.17)$ & $<0.001$ \\
\hline
\end{tabular}

Values are reported in medians (interquartile range: $\mathrm{P} 25$ to $\mathrm{P} 75$ ).

$\mathrm{CA}=$ coronary angiography $\mathrm{CD}=$ cumulative dose DAP $=$ dose area product; LRA $=$ left radial access; $\mathrm{PCI}=$ percutaneous coronary intervention; RFA = right femoral access; RRA = right radial access; $\mathrm{SD}=$ standard deviation.

to initial scientific concerns and despite the operator's inconvenience when leaning over to reach the patient's left side. To our knowledge, this is the first single-center trial to compare operator radiation exposure among these 3 vascular access sites for CA and CA followed by ad hoc PCI. Recently, the Randomized Evaluation of Vascular Entry Site and Radiation Exposure trial ${ }^{14}$ evaluated patient and operator radiation exposure among the 3 access sites RFA, LRA, and RRA and included only CA procedures without CA followed by ad hoc PCI procedures. It reported higher operator radiation for LRA using air kerma as primary end point, which is not the ideal patient exposure metric, although also analyzing DAP as a secondary end point and found an FT much shorter than our study's, presumably because PCIs were excluded. Furthermore, the population included in the Randomized Evaluation of Vascular Entry Site and Radiation Exposure trial shows a median weight and height of 64 to $65 \mathrm{~kg}$ and 163 to $164 \mathrm{~cm}$, respectively, resulting subsequently in lower DAP values (in the order of $26 \mathrm{Gycm}^{2}$ per procedure).

Both procedure-related and operator-related factors appear responsible for the differences in operator radiation exposure per vascular access site. It has been reported that increased operator radiation exposure during radial access, as opposed to femoral access, is related to increase in FT, reflecting technical difficulties and to the slightly closer operator's position to the x-ray tube and to the patient, compared with femoral access. ${ }^{5,15,16}$ In contrast, decreased operator radiation dose and shorter FT have been reported when using LRA compared with RRA. ${ }^{10,11,17-20}$ In our study, there are some details in radiation protection techniques that should be taken into consideration: First, during RRA procedures, the leaded glass mobile panel is positioned less proximally to the table compared with LRA and RFA to facilitate the right radial access. The radioprotection gap which is inevitably created between the leaded glass mobile panel and the patient table is more pronounced during RRA procedures, thus creating a considerable source of radiation exposure to the operator (Figure 1). Second, when using RRA, the operator is positioned closer to the $\mathrm{x}$-ray tube and closer to the patient compared with both LRA and RFA procedures, increasing the effect of the patient as the main source of scatter radiation to the operator. Furthermore, technical challenges in maneuvering catheters into the coronary vessels can lead in longer FT. In particular, the vascular anatomy associated with the right radial artery, including the right subclavian artery-common brachiocephalic trunk bifurcation and the common brachiocephalic trunk-aorta bifurcation, could account for tortuosity and calcifications that could impair procedural success. In considering the left radial artery, the left subclavian artery stems directly from the aortic arch, thus reducing the technical challenges in catheter manipulation, whereas in the right femoral artery there is no such issue at all. Finally, further technical difficulties associated with radial artery access include spasm or tortuosity of the radial artery, which could increase fluoroscopy and procedure times. Nonetheless, in our study, FT did not differ significantly among the 3 access sites, suggesting a high level of operator experience across the 3 groups. On the contrary, the longer procedural times and the greater $\mathrm{NC}$ in the femoral group reflect a higher complexity of procedures being performed through this access, while still benefitting from decreased operator radiation exposure compared with the 2 radial groups.

The present study confirms the greater radiation exposure of the operator compared with the first assistant who is standing at his right side, regardless of the vascular access site. This is expected because of the nurse's greater distance from the source of radiation and from the patient. Most authors studying operator radiation dose issues report results normalized per procedure and not per-patient radiation dose measured as DAP. This means that whether the dose is higher for femoral or radial approach will be a result of both the higher or lower DAP (and FT), the distance effect, and radiation protection aspects. If the dose is normalized per DAP, one can isolate, to a certain extent, the first 2 issues, and the results will then be mostly related to the radiation protection level and the distance between operator and patient. ${ }^{21}$ Our results in terms of procedure (fluoroscopy and procedural times) and radiation dose of the operator (CD) and the patient (DAP) are in line with published data in the literature. Although not randomized, the present study reflects operator radiation exposure in a real-world population in everyday clinical practice, despite a bias in the choice of vascular access site by the operator. Furthermore, the 3 groups were homogenous with regard to characteristics that influence operator and patient irradiation such as patients' body mass index and procedural and FTs, thus reflecting a representative sample of a real-world population without exclusion of emergent or complex procedures. The greater NC in the RFA group compared with the LRA and RRA groups and in the RRA compared with the LRA group probably suggests increased procedural complexity and could be reflected in the higher patient irradiation (DAP) in the RFA group compared with the 2 radial groups. The normalization, however, of the operator irradiation for patient irradiation takes into consideration such parameters as procedural complexity and patient corpulence, thus accurately reflecting the actual operator radiation dose, and is dependent only on the following 2 parameters: (1) the degree of radioprotection itself and (2) the distance between patient and operator. Therefore, interpretation of results using the DAP-normalized operator radiation 
dose allows for isolation and meaningful evaluation of technical differences among the 3 vascular access sites based on the positioning of radioprotection equipment, the operator's position, and his distance from the patient and the $\mathrm{x}$-ray tube.

This was a nonrandomized, single-center study. Therefore, these results have to be interpreted with caution for other catheterization laboratories because of possible differences in operator experience, training and techniques, radioprotection materials, and devices. Furthermore, procedures were performed by 5 different, although highly experienced, interventional cardiologists implicating differences in catheterization techniques, procedural and FTs, $\mathrm{NC}$, and thus effecting individual radiation dose. Finally, in our study, operator dose was measured by 1 single dosimeter positioned externally to the sternum, with no possibility to measure and compare radiation exposure of other corporal areas.

\section{Disclosures}

The authors have no conflicts of interest to disclose.

1. Bashore TM. Radiation safety in the cardiac catheterization laboratory Am Heart J 2004;147:375-378.

2. von Boetticher H, Lachmund J, Hoffmann W. An analytic approach to double dosimetry algorithms in occupational dosimetry using energy dependent organ dose conversion coefficients. Health Phys 2010;99: 800-805.

3. Kuon E. Radiation exposure in invasive cardiology. Heart 2008;94: 667-674.

4. Lange HW, von Boetticher H. Randomized comparison of operator radiation exposure during coronary angiography and intervention by radial or femoral approach. Catheter Cardiovasc Interv 2006;67:12-16.

5. Brasselet C, Blanpain T, Tassan-Mangina S, Deschildre A, Duval S, Vitry F, Gaillot-Petit N, Clement JP, Metz D. Comparison of operator radiation exposure with optimized radiation protection devices during coronary angiograms and ad hoc percutaneous coronary interventions by radial and femoral routes. Eur Heart J 2008;29:63-70.

6. Lange HW, von Boetticher H. Reduction of operator radiation dose by a pelvic lead shield during cardiac catheterization by radial access: comparison with femoral access. JACC Cardiovasc Interv 2012;5: 445-449.

7. Michael TT, Alomar M, Papayannis A, Mogabgab O, Patel VG, Rangan BV, Luna M, Hastings JL, Grodin J, Abdullah S, Banerjee S, Brilakis ES. A randomized comparison of the transradial and transfemoral approaches for coronary artery bypass graft angiography and intervention: the RADIAL-CABG Trial (RADIAL versus Femoral Access for Coronary Artery Bypass Graft Angiography and Intervention). JACC Cardiovasc Interv 2013;6:1138-1144.

8. Neill J, Douglas H, Richardson G, Chew EW, Walsh S, Hanratty C, Herity N. Comparison of radiation dose and the effect of operator experience in femoral and radial arterial access for coronary procedures. Am J Cardiol 2010;106:936-940.
9. Mercuri M, Mehta S, Xie C, Valettas N, Velianou JL, Natarajan MK Radial artery access as a predictor of increased radiation exposure during a diagnostic cardiac catheterization procedure. JACC Cardiovasc Interv 2011;4:347-352.

10. Kado H, Patel AM, Suryadevara S, Zenni MM, Box LC, Angiolillo DJ, Bass TA, Guzman LA. Operator radiation exposure and physical discomfort during a right versus left radial approach for coronary interventions: a randomized evaluation. JACC Cardiovasc Interv 2014;7: 810-816.

11. Dominici M, Diletti R, Milici C, Bock C, Placanica A, D’Alessandro G, Arrivi A, Italiani M, Buono E, Boschetti E. Operator exposure to $\mathrm{X}$-ray in left and right radial access during percutaneous coronary procedures: OPERA randomised study. Heart 2013;99:480-484.

12. Sciahbasi A, Romagnoli E, Trani C, Burzotta F, Sarandrea A, Summaria F, Patrizi R, Rao S, Lioy E. Operator radiation exposure during percutaneous coronary procedures through the left or right radial approach: the TALENT dosimetric substudy. Circ Cardiovasc Interv 2011;4:226-231.

13. Kanei Y, Nakra NC, Liou M, Vales LL, Gowda R, Rosero H, Kwan T, Fox JT. Randomized comparison of transradial coronary angiography via right or left radial artery approaches. Am J Cardiol 2011;107: 195-197.

14. Pancholy SB, Joshi P, Shah S, Rao SV, Bertrand OF, Patel TM. Effect of vascular access site choice on radiation exposure during coronary angiography: the REVERE trial (Randomized Evaluation of Vascular Entry Site and Radiation Exposure). JACC Cardiovasc Interv 2015;8: 1189-1196.

15. Kiemeneij F, Laarman GJ, Odekerken D, Slagboom T, van der Wieken $\mathrm{R}$. A randomized comparison of percutaneous transluminal coronary angioplasty by the radial, brachial and femoral approaches: the access study. J Am Coll Cardiol 1997;29:1269-1275.

16. Vano E. Radiation exposure to cardiologists: how it could be reduced. Heart 2003;89:1123-1124.

17. Pelliccia F, Trani C, Biondi-Zoccai GG, Nazzaro M, Berni A, Patti G, Patrizi R, Pironi B, Mazzarotto P, Gioffre G, Speciale G, Pristipino C; Prospective Registry of Vascular Access in Interventions in Lazio Region Study Group. Comparison of the feasibility and effectiveness of transradial coronary angiography via right versus left radial artery approaches (from the PREVAIL Study). Am J Cardiol 2012;110: $771-775$.

18. Freixa X, Trilla M, Feldman M, Jimenez M, Betriu A, Masotti M Right versus left transradial approach for coronary catheterization in octogenarian patients. Catheter Cardiovasc Interv 2012;80:267-272.

19. Larsen P, Shah S, Waxman S, Freilich M, Riskalla N, Piemonte T, Jeon C, Pyne C. Comparison of procedural times, success rates, and safety between left versus right radial arterial access in primary percutaneous coronary intervention for acute ST-segment elevation myocardial infarction. Catheter Cardiovasc Interv 2011;78:38-44.

20. Biondi-Zoccai G, Sciahbasi A, Bodi V, Fernandez-Portales J, Kanei Y, Romagnoli E, Agostoni P, Sangiorgi G, Lotrionte M, Modena MG. Right versus left radial artery access for coronary procedures: an international collaborative systematic review and meta-analysis including 5 randomized trials and 3210 patients. Int J Cardiol 2013;166: $621-626$.

21. Carnicer A, Baechler S, Canetti L, Verdun FR. Staff exposure in interventional cardiology for the Swiss Federal Office of Public Health, 2014. 